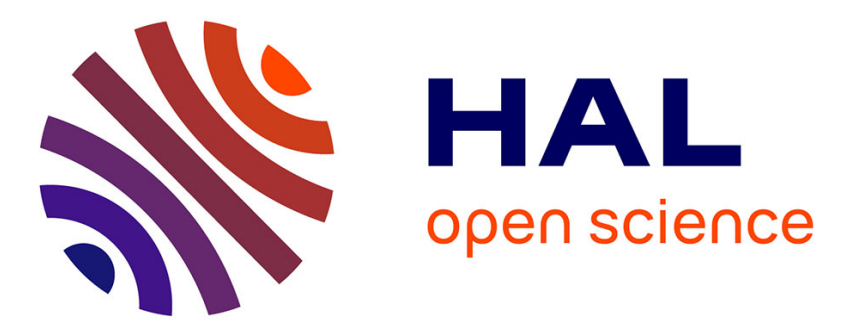

\title{
Revealing some unexpected dependence properties of linear combinations of stable random variables using symmetric covaration
}

\author{
Ludovic d'Estampes, Bernard Garel, Dag Tjøstheim
}

\section{To cite this version:}

Ludovic d'Estampes, Bernard Garel, Dag Tjøstheim. Revealing some unexpected dependence properties of linear combinations of stable random variables using symmetric covaration. Communications in Statistics - Theory and Methods, 2004, 33 (4), 10.1081/STA-120028725 . hal-01330072

\section{HAL Id: hal-01330072 \\ https://hal.science/hal-01330072}

Submitted on 9 Jun 2016

HAL is a multi-disciplinary open access archive for the deposit and dissemination of scientific research documents, whether they are published or not. The documents may come from teaching and research institutions in France or abroad, or from public or private research centers.
L'archive ouverte pluridisciplinaire HAL, est destinée au dépôt et à la diffusion de documents scientifiques de niveau recherche, publiés ou non, émanant des établissements d'enseignement et de recherche français ou étrangers, des laboratoires publics ou privés. 


\section{REVEALING SOME UNEXPECTED DEPENDENCE PROPERTIES OF LINEAR COMBINATIONS OF STABLE RANDOM VARIABLES USING SYMMETRIC COVARIATION}

Ludovic d'Estampes ${ }^{\mathrm{a}}$, Bernard Garel ${ }^{\mathrm{a}}$ and Dag Tjøstheim ${ }^{\mathrm{b}}$

aStochastic Models, ENSEEIHT, B.P.7122,

2, rue Camichel 31071 Toulouse Cedex 7, France.

garel@enseeiht.fr

${ }^{\mathrm{b}}$ Department of Mathematics, University of Bergen,

Johs. Brunsgt. 12, N-5008 Bergen, Norway.

Key Words: dependence measure; covariation; rank; correlation; $\alpha$-stable; infinite variance.

\section{ABSTRACT}

The covariation is one of the possible dependence measures for variables where distribution is symmetric alpha-stable with parameter alpha between one and two. We introduce a symmetrized and normalized version of the covariation which enables us to reveal some unexpected dependence properties of stable variables.

\section{INTRODUCTION}

In many practical situations of interest in statistics, the observed data cannot be adequately described by a Gaussian distribution. Usually in such cases a distribution with heavier tails is needed, particularly in the fields of telecommunications and finance. From a theoretical point of view, the class of stable distributions is the most satisfying heavy-tail generalization. It contains the Gaussian distribution as a special case, and it retains at least some of its properties. For example, it is infinitely divisible, linear combinations of stable variables are still stable and the conditional expectation, granted that it exists, of a stable variable $X$ with respect to another variable $Y$ is still a linear function of $Y$. Moreover an extensive asymptotic theory has been developed (see Samorodnitsky and Taqqu (1)). 
A main problem with non-Gaussian models has been the complexity of calculations, but recent developments of data processing techniques have made both model fitting with stable distributions and simulation of stable variables practical. On the other hand, because of the infinite variance of these distributions, the much applied correlation tool for assessing dependence cannot be used. So, other dependence measures are needed using moments of orders less than two.

Samorodnitsky and Taqqu (1) in chapter 4 of their book discuss several alternatives that have been used. Most of the interest seems to center around the so-called covariation measure. This measure was treated in the early papers by Miller (2) and Cambanis and Miller (3). Since then there has been a number of papers with many of the results summed up in Nikias and Shao (4). A recent alternative contribution is Pinkse (5) which introduced a non parametric serial dependence measure with the help of the characteristic function. General measures of dependence and resulting tests of independence are surveyed by Tjøstheim (6). Further developments can be found in Hong (7) and Hong and White (8).

In this paper we will focus on dependence properties of linear combinations of stable variables. Our main tool is introduced in section 2. It is a normalized and symmetrized version of the covariance measure. We use it to reveal some unexpected dependence properties in section 3. For instance, it will be shown that linear combinations exist that display both positive and negative dependence at the same time. Finally, in section 4, we present some finite sample results for the new symmetrized covariation measure and compare it to some other standard measures.

\section{COVARIATION AND SYMMETRIC COVARIATION}

We start by giving some basic properties of symmetric alpha-stable distributions.

Definition 1 A vector $\underline{X}=\left(X_{1}, \ldots, X_{n}\right)$ has a symmetric alpha-stable $(S \alpha S)$ distribution if and only if (iff) its characteristic function $\varphi$ is given by

$$
\forall\left(t_{1}, \ldots, t_{n}\right) \in \mathbb{R}^{n}, \varphi_{\left(X_{1}, \ldots, X_{n}\right)}\left(t_{1}, \ldots, t_{n}\right)=\exp \left\{-\int_{S^{n-1}}|\langle\underline{t}, \underline{x}\rangle|^{\alpha} d \mu_{S^{n-1}}(\underline{x})\right\}
$$


where $\left\{\begin{array}{l}\mu_{S^{n-1}} \text { is a symmetric measure, called the spectral measure, on the } \\ \text { Borel sets of the unit sphere } S^{n-1}=\left\{\left(x_{1}, \ldots, x_{n}\right) \in \mathbb{R}^{n} / \sum_{i=1}^{n} x_{i}^{2}=1\right\} \\ \langle\cdot, \cdot\rangle \text { represents the usual scalar product on } \mathbb{R}^{n}, \\ \alpha \in] 0 ; 2] ; \underline{t}=\left(t_{1}, \ldots, t_{n}\right) ; \underline{x}=\left(x_{1}, \ldots, x_{n}\right) .\end{array}\right.$

Definition 2 A real random variable $X$ has a $S \alpha S$ distribution, with parameters $(\gamma, \alpha)$ where $\gamma>0$ is called the dispersion of $X$, iff its characteristic function is given by

$$
\forall t \in \mathbb{R}, \varphi_{X}(t)=\exp \left\{-\gamma|t|^{\alpha}\right\} .
$$

\section{Remarks}

1. If $\underline{X}$ is a $S \alpha S$ vector, then every component $X_{i}$ is a $S \alpha S$ random variable with parameters $\left(\gamma_{X_{i}}, \alpha\right)$. We have

$$
\gamma_{X_{i}}=\int_{S^{n-1}}\left|x_{i}\right|^{\alpha} d \mu_{S^{n-1}}(\underline{x}) \text { and } \varphi_{X_{i}}\left(t_{i}\right)=\varphi_{\left(X_{1}, \ldots, X_{n}\right)}\left(0, \ldots, 0, t_{i}, 0 \ldots, 0\right) .
$$

2. The random vector $\underline{X}$ is a $S \alpha S$ random vector iff all linear combinations $\sum_{j=1}^{n} a_{j} X_{j}$ is a $S \alpha S$ random variable.

3. If $X_{1}$ and $X_{2}$ are two independent $S \alpha S$ random variables, then $\left(X_{1}, X_{2}\right)$ is a $S \alpha S$ random vector.

The following proposition follows directly from point 2 of the preceding remarks.

Proposition 3 Let $\underline{X}$ be a $S \alpha S$ random vector and $M$ a $m \times n$ real matrix. If $\underline{Y}=M \underline{X}$, then $\underline{Y}$ is a $S \alpha S$ random vector.

For the rest of the paper, we assume that $\alpha$ is greater than 1 . The covariation generalizes the covariance to the situation where there are no second moments.

Definition 4 Let $\left(Y_{1}, Y_{2}\right)$ be a $S \alpha S$ random vector, the covariation between $Y_{1}$ and $Y_{2}$ is equal to

$$
\left[Y_{1}, Y_{2}\right]_{\alpha}=\int_{S^{1}} y_{1} y_{2}^{\langle\alpha-1\rangle} d \mu_{S^{1}}\left(y_{1}, y_{2}\right)
$$


where $\mu_{S^{1}}$ is the spectral measure on the unit sphere $S^{1}$ and where we have used the notation

$$
u^{\langle v\rangle}=\operatorname{sign}(u) \cdot|u|^{v}
$$

Moreover, the coefficient of covariation of $Y_{1}$ on $Y_{2}$, is the quantity:

$$
\left\{Y_{1}, Y_{2}\right\}_{\alpha}=\frac{\left[Y_{1}, Y_{2}\right]_{\alpha}}{\left[Y_{2}, Y_{2}\right]_{\alpha}}
$$

For $\alpha=2,\left[Y_{1}, Y_{2}\right]_{2}=\frac{1}{2} \operatorname{Cov}\left(Y_{1}, Y_{2}\right)$. Note that $[Y, Y]_{\alpha}=\gamma_{Y}$.

Properties of the covariation are given in Samorodnitsky and Taqqu (1),pp. 63-64. Note that if $X$ and $Y$ are two independent $S \alpha S$ random variables then $[X, Y]_{\alpha}=0$. Furthermore, for $(a, b) \in \mathbb{R}^{2},[a X, b Y]_{\alpha}=a b^{\alpha}[X, Y]_{\alpha}$, from which it follows that for $c \neq 0$,

$$
\{c X, X\}_{\alpha}=\frac{[c X, X]_{\alpha}}{[X, X]_{\alpha}}=c \text { and }\{X, c X\}_{\alpha}=\frac{[X, c X]_{\alpha}}{[c X, c X]_{\alpha}}=\frac{1}{c} .
$$

The two preceding equalities demonstrate the well-known fact that the coefficient of covariation is not symmetric and is not bounded. Using theorem 4.1.2 of Samorodnitsky and Taqqu (1),p. 175, we have for the conditional expectation

$$
\mathbb{E}\left(Y_{2} \mid Y_{1}\right)=\left\{Y_{2}, Y_{1}\right\}_{\alpha} Y_{1}
$$

so that in general $\mathbb{E}\left(Y_{2} \mid Y_{1}\right) \neq \mathbb{E}\left(Y_{1} \mid Y_{2}\right)$. In the Gaussian case $(\alpha=2)$ we also have $\mathbb{E}\left(Y_{2} \mid Y_{1}\right) \neq \mathbb{E}\left(Y_{1} \mid Y_{2}\right)$ unless $\operatorname{Var}\left(Y_{1}\right)=\operatorname{Var}\left(Y_{2}\right)$. But the dependence is unambiguous in the sense that $\rho\left(Y_{1}, Y_{2}\right)=\rho\left(Y_{2}, Y_{1}\right)$ where $\rho$ denotes ordinary correlation and the regression line $\mathbb{E}\left(Y_{1} \mid Y_{2}\right)$ has a positive slope iff $\mathbb{E}\left(Y_{2} \mid Y_{1}\right)$ has a positive slope. It will be shown in section 3 that for stable variables with $\alpha<2$, the dependence is ambiguous. It is possible to find examples where the slope of $\mathbb{E}\left(Y_{2} \mid Y_{1}\right)$ is positive $\left(\left\{Y_{2}, Y_{1}\right\}_{\alpha}>0\right)$, whereas the slope of $\mathbb{E}\left(Y_{1} \mid Y_{2}\right)$ is negative $\left(\left\{Y_{1}, Y_{2}\right\}_{\alpha}<0\right)$. An example is given in the following scatter diagram. 
Figure 1: Plot of 50 realizations of a bivariate $S \alpha S$ random vector with $\alpha=1.5$. The theoretical regression line $\mathbb{E}\left(Y_{1} \mid Y_{2}\right)$ and $\mathbb{E}\left(Y_{2} \mid Y_{1}\right)$ are drawn in. This example comes from the system (3.1) with the following parameters: $\gamma_{X_{1}}=3 ; \gamma_{X_{2}}=2 ; a_{1}=2^{(4 / 3)} ; a_{2}=1 ; a_{3}=$ $1 ; a_{4}=-3^{(4 / 3)}$.

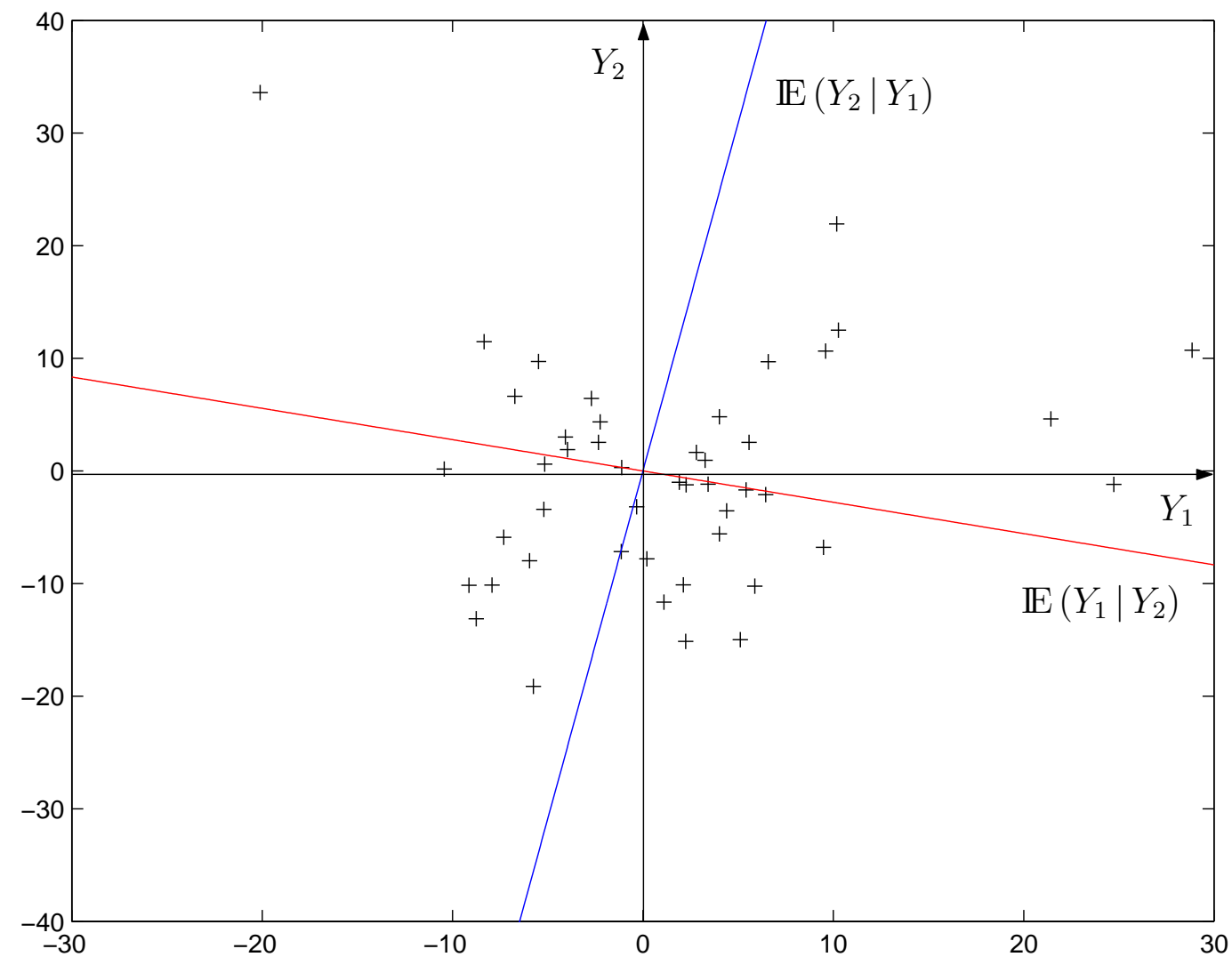

An important instrument needed to derive the results of the next section is the symmetric coefficient of covariation:

Definition 5 Let $\left(Y_{1}, Y_{2}\right)$ be a $S \alpha S$ random vector. The symmetric coefficient of covariation between $Y_{1}$ and $Y_{2}$ is equal to

$$
\operatorname{Corr}_{\alpha}\left(Y_{1}, Y_{2}\right)=\left\{Y_{1}, Y_{2}\right\}_{\alpha}\left\{Y_{2}, Y_{1}\right\}_{\alpha}=\frac{\left[Y_{1}, Y_{2}\right]_{\alpha}\left[Y_{2}, Y_{1}\right]_{\alpha}}{\left[Y_{1}, Y_{1}\right]_{\alpha}\left[Y_{2}, Y_{2}\right]_{\alpha}}
$$


In the Gaussian case (for $\alpha=2)$, $\operatorname{Corr}_{\alpha}\left(Y_{1}, Y_{2}\right)=\rho^{2}\left(Y_{1}, Y_{2}\right)$, so that the symmetric coefficient of covariation is only measuring the magnitude of the dependence not its direction. One would perhaps expect that $\operatorname{Corr}_{\alpha}\left(Y_{1}, Y_{2}\right) \geqslant 0$ also for $\alpha<2$, but it turns out that we may have $\operatorname{Corr}_{\alpha}\left(Y_{1}, Y_{2}\right)<0$, meaning that $\left[Y_{1}, Y_{2}\right]_{\alpha}$ and $\left[Y_{2}, Y_{1}\right]_{\alpha}$ may have different signs, one indicating positive dependence, the other negative. Hence $\operatorname{Corr}_{\alpha}\left(Y_{1}, Y_{2}\right)$ does not only have the capability of measuring the strength of what we may call concordant dependence, but also the degree of disconcordant dependence between two variables $Y_{1}$ and $Y_{2}$.

Before demonstrating this we show that $\operatorname{Corr}_{\alpha}\left(Y_{1}, Y_{2}\right)$ is bounded, taking values between -1 and 1.

Proposition 6 Let $\left(Y_{1}, Y_{2}\right)$ be a $S \alpha S$ random vector. We have

1- $\left|\operatorname{Corr}_{\alpha}\left(Y_{1}, Y_{2}\right)\right| \leqslant 1$.

2- If $Y_{1}$ and $Y_{2}$ are two independent $S \alpha S$ random variables, then $\operatorname{Corr}_{\alpha}\left(Y_{1}, Y_{2}\right)=0$.

\section{PROOF}

1- We know that (see Samorodnitsky and Taqqu (1), Property 2.8.4, p.96)

$$
\begin{aligned}
\left|\left[Y_{1}, Y_{2}\right]_{\alpha}\right| & \leqslant\left\|Y_{1}\right\|_{\alpha}\left\|Y_{2}\right\|_{\alpha}^{\alpha-1}, \\
\text { and }\left|\left[Y_{2}, Y_{1}\right]_{\alpha}\right| & \leqslant\left\|Y_{2}\right\|_{\alpha}\left\|Y_{1}\right\|_{\alpha}^{\alpha-1} \text { where }\|U\|_{\alpha}=\left([U, U]_{\alpha}\right)^{1 / \alpha}
\end{aligned}
$$

So $\left|\left[Y_{1}, Y_{2}\right]_{\alpha}\right| \times\left|\left[Y_{2}, Y_{1}\right]_{\alpha}\right| \leqslant\left\|Y_{1}\right\|_{\alpha}\left\|Y_{2}\right\|_{\alpha}^{\alpha-1} \times\left\|Y_{2}\right\|_{\alpha}\left\|Y_{1}\right\|_{\alpha}^{\alpha-1}$

which entails that $\left|\frac{\left[Y_{1}, Y_{2}\right]_{\alpha}\left[Y_{2}, Y_{1}\right]_{\alpha}}{\left[Y_{1}, Y_{1}\right]_{\alpha}\left[Y_{2}, Y_{2}\right]_{\alpha}}\right| \leqslant 1$.

2- We know that $\left[Y_{1}, Y_{2}\right]_{\alpha}$ is equal to zero. Because the denominator is different from 0 , we obtain $\operatorname{Corr}_{\alpha}\left(Y_{1}, Y_{2}\right)=\frac{\left[Y_{1}, Y_{2}\right]_{\alpha}\left[Y_{2}, Y_{1}\right]_{\alpha}}{\left[Y_{1}, Y_{1}\right]_{\alpha}\left[Y_{2}, Y_{2}\right]_{\alpha}}=0$.

A value of $\operatorname{Corr}_{\alpha}\left(Y_{1}, Y_{2}\right)=-1$ will indicate a very high degree of disconcordance in the dependence relationship between $Y_{1}$ and $Y_{2}$. It will be seen next that such a value is possible as a limiting case. 


\section{SOME UNEXPECTED DEPENDENCE PROPERTIES OF LINEAR COMBINATIONS}

\section{OF STABLE VARIABLES}

Let $X_{1}$ and $X_{2}$ be independent $S \alpha S$ variables with $1<\alpha \leqslant 2$ and let

$$
Y_{1}=a_{1} X_{1}+a_{2} X_{2} \text { and } Y_{2}=a_{3} X_{1}+a_{4} X_{2}
$$

In the Gaussian case $(\alpha=2)$, we have

$$
\mathbb{E}\left(Y_{1} Y_{2}\right)=a_{1} a_{3} \mathbb{E}\left(X_{1}^{2}\right)+a_{2} a_{4} \mathbb{E}\left(X_{2}^{2}\right)+\left(a_{1} a_{4}+a_{2} a_{3}\right) \mathbb{E}\left(X_{1} X_{2}\right)
$$

With no restriction we assume that $\mathbb{E}\left(X_{1}^{2}\right)=\mathbb{E}\left(X_{2}^{2}\right)$. It is seen that $Y_{1}$ and $Y_{2}$ are independent iff $a_{1} a_{3}=-a_{2} a_{4}$. In this case, $\mathbb{E}\left(Y_{1} \mid Y_{2}\right)=\mathbb{E}\left(Y_{2} \mid Y_{1}\right)=0$. However, as will be seen in the next proposition, such linear combinations in general yields $\operatorname{Corr}_{\alpha}\left(Y_{1}, Y_{2}\right)<0$. By (2.1) and (2.2), this means that if $Y_{2}$ increases with $Y_{1}$ in the regression of $Y_{2}$ on $Y_{1}$, then $Y_{1}$ decreases with increasing $Y_{2}$ in the regression of $Y_{1}$ on $Y_{2}$. Again, mainly to ease notation, we assume $\left[X_{1}, X_{1}\right]_{\alpha}=\gamma_{X_{1}}=\gamma_{X_{2}}=\left[X_{2}, X_{2}\right]_{\alpha}$, which corresponds to $\mathbb{E}\left(X_{1}^{2}\right)=\mathbb{E}\left(X_{2}^{2}\right)$ in the Gaussian case.

Proposition 7 Consider the system (3.1) for $1<\alpha \leqslant 2$. Then

$$
\begin{aligned}
\operatorname{Corr}_{\alpha}\left(Y_{1}, Y_{2}\right) & =\frac{a_{1} a_{3} \operatorname{sign}\left(a_{1} a_{3}\right)\left|a_{1} a_{3}\right|^{\alpha-1}+a_{2} a_{4} \operatorname{sign}\left(a_{2} a_{4}\right)\left|a_{2} a_{4}\right|^{\alpha-1}}{\left(\left|a_{3}\right|^{\alpha}+\left|a_{4}\right|^{\alpha}\right)\left(\left|a_{1}\right|^{\alpha}+\left|a_{2}\right|^{\alpha}\right)} \\
& +\frac{a_{1} a_{4} \operatorname{sign}\left(a_{2} a_{3}\right)\left|a_{2} a_{3}\right|^{\alpha-1}+a_{2} a_{3} \operatorname{sign}\left(a_{1} a_{4}\right)\left|a_{1} a_{4}\right|^{\alpha-1}}{\left(\left|a_{3}\right|^{\alpha}+\left|a_{4}\right|^{\alpha}\right)\left(\left|a_{1}\right|^{\alpha}+\left|a_{2}\right|^{\alpha}\right)} .
\end{aligned}
$$

If $a_{1} a_{3}=-a_{2} a_{4}$, this simplifies to

$$
\operatorname{Corr}_{\alpha}\left(Y_{1}, Y_{2}\right)=-\frac{\left(a_{1} a_{3}\right)^{2}\left\{\left|a_{2} a_{3}\right|^{\frac{\alpha}{2}-1}-\left|a_{1} a_{4}\right|^{\frac{\alpha}{2}-1}\right\}^{2}}{\left(\left|a_{1}\right|^{\alpha}+\left|a_{2}\right|^{\alpha}\right)\left(\left|a_{3}\right|^{\alpha}+\left|a_{4}\right|^{\alpha}\right)}
$$

which is negative.

\section{PROOF}

Using independence between $X_{1}$ and $X_{2}$ in (3.1), we have

$$
\operatorname{Corr}_{\alpha}\left(Y_{1}, Y_{2}\right)=\frac{a_{1} a_{3}^{\langle\alpha-1\rangle}+a_{2} a_{4}^{\langle\alpha-1\rangle}}{\left|a_{3}\right|^{\alpha}+\left|a_{4}\right|^{\alpha}} \times \frac{a_{3} a_{1}^{\langle\alpha-1\rangle}+a_{4} a_{2}^{\langle\alpha-1\rangle}}{\left|a_{1}\right|^{\alpha}+\left|a_{2}\right|^{\alpha}} .
$$


Using the definition of $u^{\langle v\rangle}$ (Definition 4, page 3), we obtain (3.2). Inserting $a_{1} a_{3}=-a_{2} a_{4}$, we have:

$$
\begin{aligned}
a_{1} a_{3} \operatorname{sign}\left(a_{1} a_{3}\right)\left|a_{1} a_{3}\right|^{\alpha-1}+a_{2} a_{4} \operatorname{sign}\left(a_{2} a_{4}\right)\left|a_{2} a_{4}\right|^{\alpha-1} & =2\left|a_{1}\right|^{\alpha}\left|a_{3}\right|^{\alpha} \\
& =2\left|a_{1}\right|^{\frac{\alpha}{2}}\left|a_{2}\right|^{\frac{\alpha}{2}}\left|a_{3}\right|^{\frac{\alpha}{2}}\left|a_{4}\right|^{\frac{\alpha}{2}} .
\end{aligned}
$$

Moreover,

$$
\begin{aligned}
a_{1} a_{4} \operatorname{sign}\left(a_{2} a_{3}\right)\left|a_{2} a_{3}\right|^{\alpha-1} & =\operatorname{sign}\left(a_{1} a_{2} a_{3} a_{4}\right)\left|a_{1}\right|\left|a_{4}\right|\left|a_{2}\right|^{\alpha-1}\left|a_{3}\right|^{\alpha-1} \\
& =-\left|a_{1}\right|\left|a_{4}\right|\left|a_{2}\right|^{\alpha-1}\left|a_{3}\right|^{\alpha-1},
\end{aligned}
$$

where $\operatorname{sign}\left(a_{1} a_{2} a_{3} a_{4}\right)=-1$ because $a_{1} a_{3}=-a_{2} a_{4}$. Similarly, we have

$$
a_{2} a_{3} \operatorname{sign}\left(a_{1} a_{4}\right)\left|a_{1} a_{4}\right|^{\alpha-1}=-\left|a_{2}\right|\left|a_{3}\right|\left|a_{1}\right|^{\alpha-1}\left|a_{4}\right|^{\alpha-1} .
$$

Inserted in (3.2), this yields

$$
\begin{aligned}
\operatorname{Corr}_{\alpha}\left(Y_{1}, Y_{2}\right)= & \frac{2\left|a_{1}\right|^{\frac{\alpha}{2}}\left|a_{2}\right|^{\frac{\alpha}{2}}\left|a_{3}\right|^{\frac{\alpha}{2}}\left|a_{4}\right|^{\frac{\alpha}{2}}}{\left(\left|a_{3}\right|^{\alpha}+\left|a_{4}\right|^{\alpha}\right)\left(\left|a_{1}\right|^{\alpha}+\left|a_{2}\right|^{\alpha}\right)} \\
& -\frac{\left|a_{1}\right|\left|a_{4}\right|\left|a_{2}\right|^{\alpha-1}\left|a_{3}\right|^{\alpha-1}+\left|a_{2}\right|\left|a_{3}\right|\left|a_{1}\right|^{\alpha-1}\left|a_{4}\right|^{\alpha-1}}{\left(\left|a_{3}\right|^{\alpha}+\left|a_{4}\right|^{\alpha}\right)\left(\left|a_{1}\right|^{\alpha}+\left|a_{2}\right|^{\alpha}\right)}, \\
\operatorname{Corr}_{\alpha}\left(Y_{1}, Y_{2}\right)= & \frac{-\left|a_{1} a_{2} a_{3} a_{4}\right|\left(\left|a_{2} a_{3}\right|^{\alpha-2}-2\left|a_{2} a_{3}\right|^{\frac{\alpha}{2}-1}\left|a_{1} a_{4}\right|^{\frac{\alpha}{2}-1}+\left|a_{1} a_{4}\right|^{\alpha-2}\right)}{\left(\left|a_{3}\right|^{\alpha}+\left|a_{4}\right|^{\alpha}\right)\left(\left|a_{1}\right|^{\alpha}+\left|a_{2}\right|^{\alpha}\right)}
\end{aligned}
$$

from which (3.3) follows by setting $\left|a_{1} a_{3}\right|=\left|a_{2} a_{4}\right|$.

It is seen from (3.3) that once $a_{1} a_{3}=-a_{2} a_{4}$ holds, $\operatorname{Corr}_{\alpha}\left(Y_{1}, Y_{2}\right)=0$ if $\alpha=2$ irrespective of the values of $a_{1}, a_{2}, a_{3}, a_{4}$. This is of course the Gaussian independence case. We have $\operatorname{Corr}_{\alpha}=0$ also if one of the $a_{i}$-s is equal to zero or if $\left|a_{2} a_{3}\right|=\left|a_{1} a_{4}\right|$. The latter case does not imply independence for $\alpha<2$ (cf Samorodnitsky and Taqqu (1), Proposition 2.9.5, p.102). In fact it follows from this proposition that for the system (3.1) we cannot have independence between $Y_{1}$ and $Y_{2}$ for $\alpha<2$ if $a_{1} a_{2} a_{3} a_{4} \neq 0$.

It is possible to obtain a maximum case of ambiguity in the dependence relationship between $Y_{1}$ and $Y_{2}$ in that $\operatorname{Corr}_{\alpha}\left(Y_{1}, Y_{2}\right) \rightarrow-1$ as $\alpha \rightarrow 1, a_{1}=a_{4} \rightarrow 0$ and $\left|a_{3}\right|=\left|a_{2}\right|$. 
In this limiting case, the regression lines $\mathbb{E}\left(Y_{2} \mid Y_{1}\right)$ and $\mathbb{E}\left(Y_{1} \mid Y_{2}\right)$ are orthogonal to each other.

It is fully possible to obtain $\operatorname{Corr}_{\alpha}\left(Y_{1}, Y_{2}\right)<0$ also when the condition $a_{1} a_{3}=-a_{2} a_{4}$ does not hold. One can also say something about how often this will occur. To see this, rewrite $\operatorname{Corr}_{\alpha}\left(Y_{1}, Y_{2}\right)$ as

$$
\begin{aligned}
\operatorname{Corr}_{\alpha}\left(Y_{1}, Y_{2}\right)= & \left(\frac{\left|a_{1}\right|\left|a_{3}\right|^{\alpha-1} \operatorname{sign}\left(a_{1} a_{3}\right)+\left|a_{2}\right|\left|a_{4}\right|^{\alpha-1} \operatorname{sign}\left(a_{2} a_{4}\right)}{\left|a_{3}\right|^{\alpha}+\left|a_{4}\right|^{\alpha}}\right) \\
& \times\left(\frac{\left|a_{3}\right|\left|a_{1}\right|^{\alpha-1} \operatorname{sign}\left(a_{1} a_{3}\right)+\left|a_{4}\right|\left|a_{2}\right|^{\alpha-1} \operatorname{sign}\left(a_{2} a_{4}\right)}{\left|a_{1}\right|^{\alpha}+\left|a_{2}\right|^{\alpha}}\right) .
\end{aligned}
$$

Next, note that there are four possible combinations for the $\operatorname{sign}\left(a_{1} a_{3}\right)$ and $\operatorname{sign}\left(a_{2} a_{4}\right)$.

(i) $\operatorname{sign}\left(a_{1} a_{3}\right)=\operatorname{sign}\left(a_{2} a_{4}\right)=+1$,

(ii) $\operatorname{sign}\left(a_{1} a_{3}\right)=\operatorname{sign}\left(a_{2} a_{4}\right)=-1$,

(iii) $\operatorname{sign}\left(a_{1} a_{3}\right)=+1 ; \operatorname{sign}\left(a_{2} a_{4}\right)=-1$,

(iv) $\operatorname{sign}\left(a_{1} a_{3}\right)=-1 ; \operatorname{sign}\left(a_{2} a_{4}\right)=+1$.

In situations (i) and (ii), we have

$$
\operatorname{Corr}_{\alpha}\left(Y_{1}, Y_{2}\right)=\frac{\left(\left|a_{1}\right|\left|a_{3}\right|^{\alpha-1}+\left|a_{2}\right|\left|a_{4}\right|^{\alpha-1}\right)\left(\left|a_{3}\right|\left|a_{1}\right|^{\alpha-1}+\left|a_{4}\right|\left|a_{2}\right|^{\alpha-1}\right)}{\left(\left|a_{3}\right|^{\alpha}+\left|a_{4}\right|^{\alpha}\right)\left(\left|a_{1}\right|^{\alpha}+\left|a_{2}\right|^{\alpha}\right)}
$$

which is positive, whereas in situations (iii) and (iv), we have

$$
\operatorname{Corr}_{\alpha}\left(Y_{1}, Y_{2}\right)=\frac{\left(\left|a_{1}\right|\left|a_{3}\right|^{\alpha-1}-\left|a_{2}\right|\left|a_{4}\right|^{\alpha-1}\right)\left(\left|a_{3}\right|\left|a_{1}\right|^{\alpha-1}-\left|a_{4}\right|\left|a_{2}\right|^{\alpha-1}\right)}{\left(\left|a_{3}\right|^{\alpha}+\left|a_{4}\right|^{\alpha}\right)\left(\left|a_{1}\right|^{\alpha}+\left|a_{2}\right|^{\alpha}\right)} .
$$

It is seen that the last expression can be negative as soon as $\alpha<2$.

If we let $\alpha \rightarrow 1$, possibilities (i) and (ii) mean that $\operatorname{Corr}_{\alpha}\left(Y_{1}, Y_{2}\right) \rightarrow 1$. On the other hand, possibilities (iii) and (iv) both imply

$$
\operatorname{Corr}_{\alpha}\left(Y_{1}, Y_{2}\right) \longrightarrow \frac{\left(\left|a_{1}\right|-\left|a_{2}\right|\right)\left(\left|a_{3}\right|-\left|a_{4}\right|\right)}{\left(\left|a_{3}\right|+\left|a_{4}\right|\right)\left(\left|a_{1}\right|+\left|a_{2}\right|\right)}
$$

Thus if the coefficients are drawn at random from uniform distributions on $[-1 ; 1]$, say, then in the long run $50 \%$ will give $\operatorname{Corr}_{\alpha}\left(Y_{1}, Y_{2}\right) \approx 1$, which is the greatest possible value of the 
symmetric coefficient of covariation. The remaining $50 \%$ will be approximately symmetrically distributed around zero, i.e. about $25 \%$ will result in an ambiguous dependence relationship. This is confirmed in the four simulation plots in Figure 2.

Figure 2: Histogram of 5000 symmetric coefficients of covariation for system (3.1) with different values of $\alpha$. The $a_{i}$-s have been drawn independently from uniform distribution on $[-1,1]$.

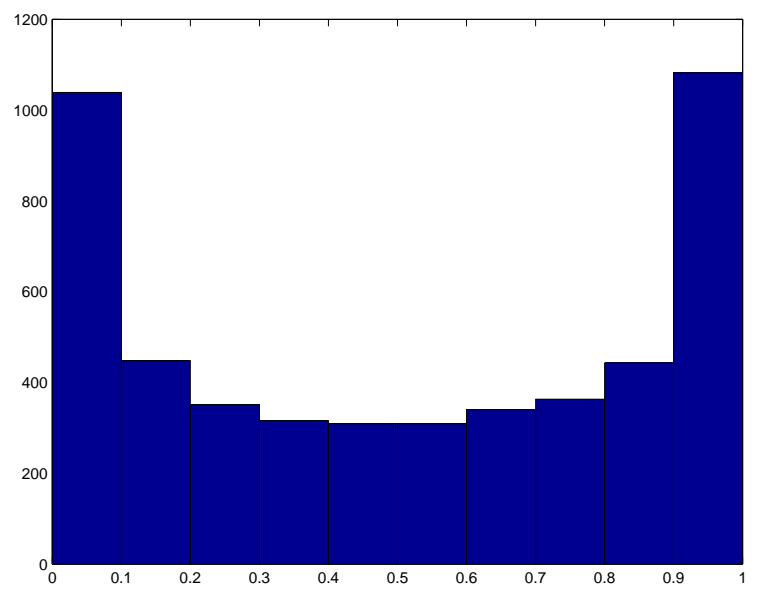

(a) $\alpha=2$

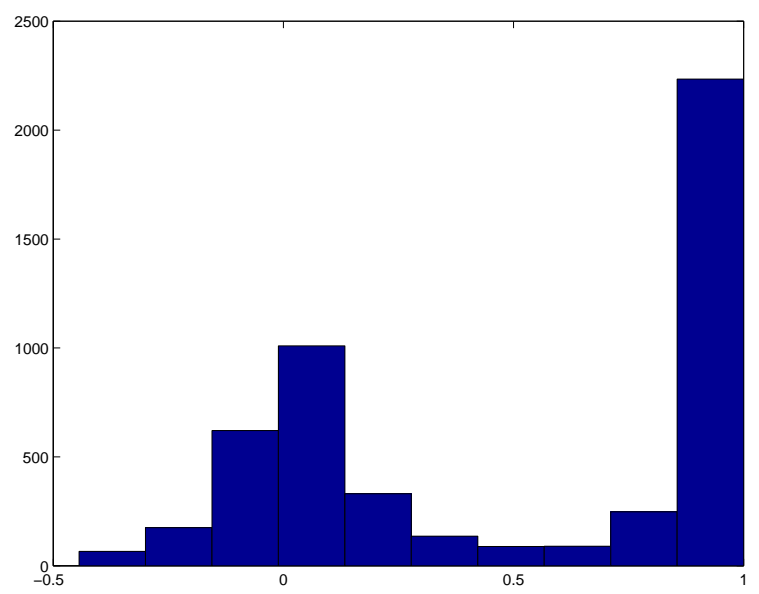

(c) $\alpha=1.1$

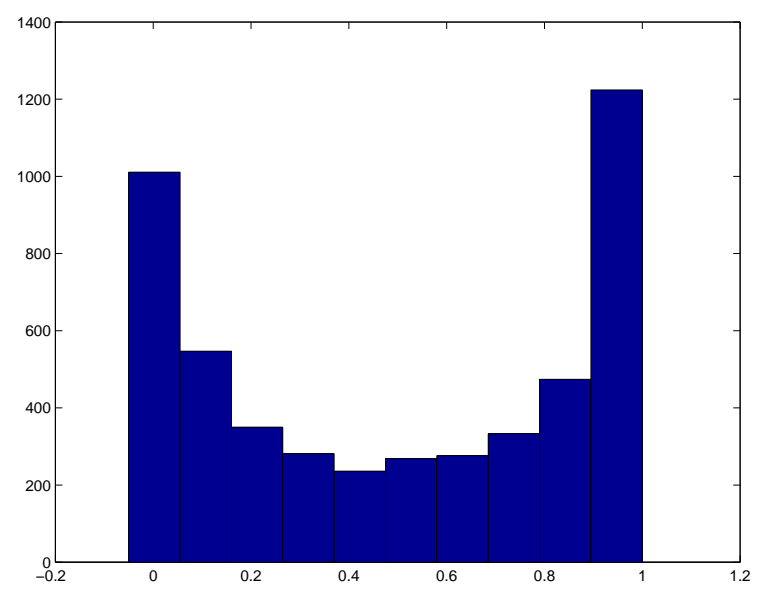

(b) $\alpha=1.5$

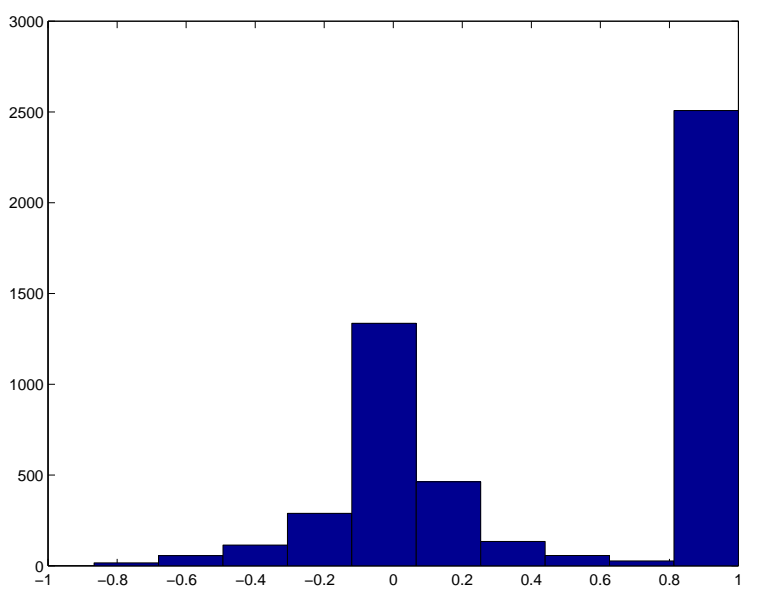

(d) $\alpha=1.01$

From the above expressions, it is easy to find values $a_{1}, a_{2}, a_{3}, a_{4}$ for which $\operatorname{Corr}_{\alpha}\left(Y_{1}, Y_{2}\right)=$ 0 . Moreover, we can also find values where $\left\{Y_{1}, Y_{2}\right\}_{\alpha}=0$ and $\left\{Y_{2}, Y_{1}\right\}_{\alpha} \neq 0$. For $\alpha<2$ 
neither of these examples would in general imply independence due to Samorodnitsky and Taqqu (1), Proposition 2.9.5, p.102.

One can also ask the question as to when $\operatorname{Corr}_{\alpha}\left(Y_{1}, Y_{2}\right)=1$.

Proposition 8 Consider the system (3.1) for $1<\alpha \leqslant 2$. Then

$$
\operatorname{Corr}_{\alpha}\left(Y_{1}, Y_{2}\right)=1 \Longleftrightarrow a_{1} a_{4}=a_{2} a_{3}
$$

\section{PROOF}

By (3.2), we have

$$
\operatorname{Corr}_{\alpha}\left(Y_{1}, Y_{2}\right)=\frac{\left|a_{1} a_{3}\right|^{\alpha}+\left|a_{2} a_{4}\right|^{\alpha}+\operatorname{sign}\left(a_{1} a_{2} a_{3} a_{4}\right)\left(\left|a_{1} a_{4}\right|\left|a_{2} a_{3}\right|^{\alpha-1}+\left|a_{2} a_{3}\right|\left|a_{1} a_{4}\right|^{\alpha-1}\right)}{\left(\left|a_{3}\right|^{\alpha}+\left|a_{4}\right|^{\alpha}\right)\left(\left|a_{1}\right|^{\alpha}+\left|a_{2}\right|^{\alpha}\right)} .
$$

Hence, $\operatorname{Corr}_{\alpha}\left(Y_{1}, Y_{2}\right)=1$ iff

$$
\operatorname{sign}\left(a_{1} a_{2} a_{3} a_{4}\right)\left(\left|a_{1} a_{4}\right|\left|a_{2} a_{3}\right|^{\alpha-1}+\left|a_{2} a_{3}\right|\left|a_{1} a_{4}\right|^{\alpha-1}\right)=\left|a_{1} a_{4}\right|^{\alpha}+\left|a_{2} a_{3}\right|^{\alpha} .
$$

At this point, there are two cases.

First case, $\operatorname{sign}\left(a_{1} a_{2} a_{3} a_{4}\right)=0$; then first we can take $a_{1}=0$. Inserting in the preceding equation, we have

$$
\left|a_{2} a_{3}\right|^{\alpha}=0 \Rightarrow a_{2} a_{3}=0
$$

In the same way, we have the three following implications

$$
\begin{aligned}
& a_{3}=0 \Rightarrow a_{1} a_{4}=0, \\
& a_{2}=0 \Rightarrow a_{1} a_{4}=0, \\
& a_{4}=0 \Rightarrow a_{2} a_{3}=0, \text { and so, we have } \operatorname{Corr}_{\alpha}\left(Y_{1}, Y_{2}\right)=1 \Leftrightarrow a_{1} a_{4}=a_{2} a_{3} .
\end{aligned}
$$

Second case, $\operatorname{sign}\left(a_{1} a_{2} a_{3} a_{4}\right) \neq 0$; then equation (3.4) is equivalent to

$$
\left[\left|a_{1} a_{4}\right|-\operatorname{sign}\left(a_{1} a_{2} a_{3} a_{4}\right)\left|a_{2} a_{3}\right|\right]\left[\left|a_{1} a_{4}\right|^{\alpha-1}-\operatorname{sign}\left(a_{1} a_{2} a_{3} a_{4}\right)\left|a_{2} a_{3}\right|^{\alpha-1}\right]=0 .
$$

If $\operatorname{sign}\left(a_{1} a_{2} a_{3} a_{4}\right)=-1$, then there is no solution.

If $\operatorname{sign}\left(a_{1} a_{2} a_{3} a_{4}\right)=+1$, then $a_{1} a_{4}=a_{2} a_{3}$. 
In the Gaussian case, $\rho^{2}\left(Y_{1}, Y_{2}\right)=\operatorname{Corr}_{\alpha}\left(Y_{1}, Y_{2}\right)=1$ is equivalent to $Y_{1}=k Y_{2}$ for some constant $k$. In the general case for $1<\alpha<2$, it is easily seen from proposition 8 that if $X_{1} \neq 0$ then $\operatorname{Corr}_{\alpha}\left(Y_{1}, Y_{2}\right)=1$ is equivalent to $a_{4} Y_{1}=a_{2} Y_{2}$, again $Y_{1}$ being a multiple of $Y_{2}$.

\section{A COMPARISON OF DEPENDENCE MEASURES}

We close this paper by examining some finite sample properties of the symmetric covariation $\operatorname{Corr}_{\alpha}\left(Y_{1}, Y_{2}\right)$ and comparing to other dependence measures for stable variables, namely the covariation $\left\{Y_{1}, Y_{2}\right\}_{\alpha}$ and two rank-based measures.

4.1. Other dependence measures

We consider two rank-based measures of dependence: the Spearman and the van der Waerden rank correlation coefficients.

Definition 9 Let $\left(X_{1}, \ldots, X_{n}\right)$ be $n$ real random variables. The associated vector of ranks, $\left(R_{1}, \ldots, R_{n}\right)$ is defined by

$$
\forall i \in \llbracket 1 ; n \rrbracket, \quad R_{i}=1+\sum_{j=1}^{n} \mathbb{1}_{] 0 ;+\infty[}\left(X_{i}-X_{j}\right)
$$

Suppose that $\left(X_{1}, Y_{1}\right), \ldots,\left(X_{n}, Y_{n}\right)$ is a sample. If we associate to $X$ (resp. $Y$ ) the vector of ranks $\left(R_{i}\right)_{i=1, \ldots, n}\left(\operatorname{resp} .\left(S_{i}\right)_{i=1, \ldots, n}\right)$, we calculate two measures of dependence.

Definition 10 With the preceding notations, the Spearman correlation coefficient $r_{S}$ and the van der Waerden correlation coefficient $r_{W}$ are given by

$$
\begin{aligned}
r_{S} & =1-\frac{6}{n\left(n^{2}-1\right)} \sum_{i=1}^{n}\left(R_{i}-S_{i}\right)^{2} \\
r_{W} & =\frac{\sum_{i=1}^{n} \Phi^{-1}\left(\frac{R_{i}}{n+1}\right) \Phi^{-1}\left(\frac{S_{i}}{n+1}\right)}{\sum_{i=1}^{n}\left[\Phi^{-1}\left(\frac{i}{n+1}\right)\right]^{2}}
\end{aligned}
$$

where $\Phi^{-1}$ is the inverse cumulative distribution function of a standard normal variable. 
These coefficients exist independently of the existence of any moments. So it is tempting to use them as a measure of dependence for alpha stable random variables. In particular, we have the following result.

Proposition 11 Under the hypothesis of independence of $X$ and $Y$, we have the asymptotic result

$$
\begin{array}{ll}
\sqrt{n-1} r_{S} & \underset{n \rightarrow+\infty}{\stackrel{\mathcal{L}}{\longrightarrow}} \mathcal{N}(0 ; 1), \\
\sqrt{n-1} r_{W} & \underset{n \rightarrow+\infty}{\stackrel{\mathcal{L}}{\longrightarrow}} \mathcal{N}(0 ; 1),
\end{array}
$$

where $\stackrel{\mathcal{L}}{\longrightarrow}$ denotes convergence in distribution.

\subsection{Simulations}

We will compare the new measure $\operatorname{Corr}_{\alpha}\left(Y_{1}, Y_{2}\right)$ to the other measures by simulations. We recall an important property of the coefficient of covariation.

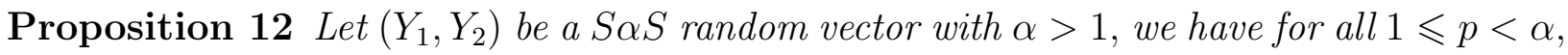

$$
\left\{Y_{1}, Y_{2}\right\}_{\alpha}=\frac{\left[Y_{1}, Y_{2}\right]_{\alpha}}{\left[Y_{2}, Y_{2}\right]_{\alpha}}=\frac{\mathbb{E}\left[Y_{1} Y_{2}^{\langle p-1\rangle}\right]}{\mathbb{E}\left|Y_{2}\right|^{p}}
$$

Samorodnitsky and Taqqu (1), pp. 94-95, gave the proof of this result for $1<p<\alpha$. Following the same lines as Samorodnitsky and Taqqu, the property is easily obtained for $p=1$. Note that this last result is used by several authors, such as Nikias and Shao (4), p.47, equation (4.4), or Gallagher (9), p.382, equation (1.5).

Taking $p=1$ in the preceding equation leads to a very convenient estimator of $\left\{Y_{1}, Y_{2}\right\}_{\alpha}$.

Let $\left(Y_{1, i}\right)_{1 \leqslant i \leqslant n}$ and $\left(Y_{2, i}\right)_{1 \leqslant i \leqslant n}$ be two vectors of realizations of $Y_{1}$ and $Y_{2}$. To estimate $\operatorname{Corr}_{\alpha}\left(Y_{1}, Y_{2}\right)$, we used the following quantity

$$
\widehat{\operatorname{Corr}_{\alpha}}\left(Y_{1}, Y_{2}\right)=\left(\frac{\sum_{i=1}^{n} Y_{1, i} \operatorname{sign}\left(Y_{2, i}\right)}{\sum_{i=1}^{n}\left|Y_{2, i}\right|}\right)\left(\frac{\sum_{i=1}^{n} Y_{2, i} \operatorname{sign}\left(Y_{1, i}\right)}{\sum_{i=1}^{n}\left|Y_{1, i}\right|}\right)
$$


which is the product of the estimator of the covariation $\left\{Y_{1}, Y_{2}\right\}_{\alpha}$ by the estimator of the covariation $\left\{Y_{2}, Y_{1}\right\}_{\alpha}$.

The algorithm of Chambers et al. (10) is used to simulate $S \alpha S$ independent random variables.

We simulated 500 realizations of $X_{1}$ and $X_{2}$, with the same dispersion $\gamma_{X_{1}}=\gamma_{X_{2}}=1$. Then, we calculated the different measures of dependence between $Y_{1}$ and $Y_{2}$ generated by (3.1). We made 100 replications. In Tables 1 and 2 there are three types of cells:

1. with a single value in the cell, it represents the theoretical value of the dependence measure (respectively the coefficient of covariation of $Y_{1}$ on $Y_{2}$, the coefficient of covariation of $Y_{2}$ on $Y_{1}$ and the symmetric coefficient of covariation between $Y_{1}$ and $Y_{2}$ );

2. with two values; these two numbers represent the mean (upper value in the cell) and the standard deviation (lower value in the cell) of the 100-sample dependence measures (respectively the Spearman correlation coefficient and the van der Waerden correlation coefficient);

3. with three values; they represent the mean (upper value in the cell), the standard deviation (center value in the cell) and the squared error (lower value in the cell) of the 100-sample dependence measures (respectively the coefficient of covariation of $Y_{1}$ on $Y_{2}$, the coefficient of covariation of $Y_{2}$ on $Y_{1}$ and the symmetric coefficient of covariation between $Y_{1}$ and $Y_{2}$ ).

We only study two sets of the four parameters $a_{1}, a_{2}, a_{3}$ and $a_{4}$. For the first case, shown in Table 1, we have independence between $Y_{1}$ and $Y_{2}$. In the second case, shown in Table 2, the parameters were drawn at random from uniform distributions, resulting in $a_{1}=0.09, a_{2}=$ $0.53, a_{3}=-0.77, a_{4}=-0.88$.

We recall that for model (3.1), we have

$$
\left\{Y_{1}, Y_{2}\right\}_{\alpha}=\frac{a_{1} a_{3}^{\langle\alpha-1\rangle}+a_{2} a_{4}^{\langle\alpha-1\rangle}}{\left|a_{3}\right|^{\alpha}+\left|a_{4}\right|^{\alpha}} .
$$


In Table 1, we see that the sample coefficient of covariation has a much bigger dispersion than the other measures when $\alpha$ goes to 1 . Both the bias and standard error vary with $\alpha$. If one looks at the squared error, none of the criteria dominates all of the others.

For the Table 2 we remark:

1. The absolute theoretical value of $\left\{Y_{1}, Y_{2}\right\}_{\alpha}$ decreases as $\alpha$ goes to 1 whereas the absolute theoretical value of $\left\{Y_{2}, Y_{1}\right\}_{\alpha}$ increases. It seems to be another drawback of this measure of dependence.

2. In the Gaussian case, the dependence between $Y_{1}$ and $Y_{2}$ is negative. The direction of dependence is detected by the two rank-based coefficients of correlation and $\left\{Y_{1}, Y_{2}\right\}_{\alpha}$ but not of course by the symmetric coefficient of covariation.

3. Even the distribution-free, rank-based coefficients of correlation vary when alpha tends to 1 . This means that there is a possible change in the dependence structure for alpha close to 1 .

We made a number of additional simulations, with different sets of parameters $\left(a_{1}, a_{2}, a_{3}, a_{4}\right)$ and different dispersions $\left(\gamma_{X_{1}}, \gamma_{X_{2}}\right)$. They gave similar results: the rank-based coefficients of correlation and the symmetric coefficient of covariation appear to be more precise in terms of squared error. 
Table I: Theoretical and estimated values of dependence measures, and rank-based coefficients of correlation for: $a_{1}=1 ; a_{2}=0 ; a_{3}=0 ; a_{4}=1$.

\begin{tabular}{|c|c|c|c|c|c|c|c|c|c|c|}
\hline$\alpha$ & 2 & 1.9 & 1.8 & 1.7 & 1.6 & 1.5 & 1.4 & 1.3 & 1.2 & 1.1 \\
\hline$\left\{Y_{1}, Y_{2}\right\}_{\alpha}$ & 0 & 0 & O & 0 & 0 & 0 & O & 0 & 0 & 0 \\
\hline$\left\{\widehat{Y_{1}, Y_{2}}\right\}_{\alpha}$ & $\begin{array}{l}0003 \\
.0575 \\
.0033 \\
\end{array}$ & $\begin{array}{c}-0.0029 \\
0.0628 \\
0.0040 \\
\end{array}$ & \begin{tabular}{|c|}
0.0092 \\
0.0767 \\
0.0060 \\
\end{tabular} & $\begin{array}{c}-0.0013 \\
0.1369 \\
0.0187 \\
\end{array}$ & $\begin{array}{c}0.0095 \\
0.1129 \\
0.0128 \\
\end{array}$ & $\begin{array}{c}0.0279 \\
0.2066 \\
0.0434 \\
\end{array}$ & \begin{tabular}{|c|}
-0.0090 \\
0.3002 \\
0.0902 \\
\end{tabular} & $\begin{array}{c}-0.0288 \\
0.3982 \\
0.1594 \\
\end{array}$ & \begin{tabular}{|c}
-0.2753 \\
3.5253 \\
12.5036 \\
\end{tabular} & $\begin{array}{c}0.0537 \\
0.5525 \\
0.3081 \\
\end{array}$ \\
\hline$\left\{Y_{2}, Y_{1}\right\}_{\alpha}$ & 0 & 0 & 0 & 0 & 0 & 0 & 0 & 0 & 0 & 0 \\
\hline$\left\{\widehat{Y_{2}, Y_{1}}\right\}_{\alpha}$ & $\begin{array}{c}0.0046 \\
0.0571 \\
0.0033 \\
\end{array}$ & $\begin{array}{l}-0.0098 \\
0.0628 \\
0.0040 \\
\end{array}$ & \begin{tabular}{|c|}
0.0049 \\
0.0938 \\
0.0088 \\
\end{tabular} & $\begin{array}{c}-0.0020 \\
0.1027 \\
0.0106 \\
\end{array}$ & $\begin{array}{c}-0.0469 \\
0.3834 \\
0.1492 \\
\end{array}$ & $\begin{array}{c}0.0064 \\
0.1850 \\
0.0343 \\
\end{array}$ & $\begin{array}{c}-0.0169 \\
0.1561 \\
0.0246 \\
\end{array}$ & \begin{tabular}{|c|}
-0.0483 \\
1.1559 \\
1.3383 \\
\end{tabular} & \begin{tabular}{|c|}
-0.1333 \\
0.9459 \\
0.9125 \\
\end{tabular} & \begin{tabular}{|l}
-0.0061 \\
0.8835 \\
0.7806 \\
\end{tabular} \\
\hline $\operatorname{Corr}_{\alpha}$ & 0 & 0 & 0 & 0 & 0 & 0 & 0 & 0 & 0 & 0 \\
\hline$\widehat{\operatorname{Corr}_{\alpha}}$ & $\begin{array}{c}0.0021 \\
0.0029 \\
0.0001 \\
\end{array}$ & $\begin{array}{c}0.0021 \\
0.0039 \\
0.0001 \\
\end{array}$ & \begin{tabular}{|c|}
0.0024 \\
0.0070 \\
0.0001 \\
\end{tabular} & $\begin{array}{c}0.0021 \\
0.0100 \\
0.0001 \\
\end{array}$ & $\begin{array}{c}0.0016 \\
0.0107 \\
0.0001 \\
\end{array}$ & $\begin{array}{c}0.0045 \\
0.0195 \\
0.0004 \\
\end{array}$ & \begin{tabular}{c|}
0.0049 \\
0.0213 \\
0.0005 \\
\end{tabular} & \begin{tabular}{|c|}
-0.0014 \\
0.0301 \\
0.0009 \\
\end{tabular} & \begin{tabular}{|c|}
0.0013 \\
0.0702 \\
0.0049 \\
\end{tabular} & \begin{tabular}{|c}
-0.0041 \\
0.0898 \\
0.0081 \\
\end{tabular} \\
\hline$r_{S}$ & $\begin{array}{c}0.0006 \\
0.0446\end{array}$ & $\begin{array}{c}-0.0048 \\
0.0460 \\
\end{array}$ & \begin{tabular}{|c|}
0.0058 \\
0.0525 \\
\end{tabular} & $\begin{array}{c}0.0017 \\
0.0423 \\
\end{array}$ & $\begin{array}{c}0.0046 \\
0.0447 \\
\end{array}$ & $\begin{array}{c}0.0009 \\
0.0451 \\
\end{array}$ & $\begin{array}{c}-0.0014 \\
0.0448 \\
\end{array}$ & $\begin{array}{c}-0.0044 \\
0.0368 \\
\end{array}$ & $\begin{array}{c}-0.0084 \\
0.0443 \\
\end{array}$ & $\begin{array}{c}0.0005 \\
0.0391 \\
\end{array}$ \\
\hline$r_{W}$ & $\begin{array}{c}-0.0010 \\
0.0471 \\
\end{array}$ & $\begin{array}{c}-0.0036 \\
0.0438 \\
\end{array}$ & $\begin{array}{c}0.0053 \\
0.0500 \\
\end{array}$ & $\begin{array}{c}-0.0007 \\
0.0422\end{array}$ & $\begin{array}{c}0.0029 \\
0.0433 \\
\end{array}$ & $\begin{array}{c}-0.0010 \\
0.0456 \\
\end{array}$ & $\begin{array}{c}-0.0019 \\
0.0469 \\
\end{array}$ & $\begin{array}{c}-0.0036 \\
0.0375 \\
\end{array}$ & $\begin{array}{c}-0.0094 \\
0.0460\end{array}$ & $\begin{array}{c}0.0009 \\
0.0393\end{array}$ \\
\hline
\end{tabular}

Table II: Theoretical and estimated values of dependence measures, and rank-based coefficients of correlation for: $a_{1}=0.09 ; a_{2}=0.53 ; a_{3}=-0.77 ; a_{4}=-0.88$.

\begin{tabular}{|c|c|c|c|c|c|c|c|c|c|c|}
\hline$\alpha$ & 2 & 1.9 & 1.8 & 1.7 & 1.6 & 1.5 & 1.4 & 1.3 & 1.2 & 1.1 \\
\hline$\left.Y_{1}, Y_{2}\right\}_{\alpha}$ & -0.3918 & -0.3902 & -0.3886 & -0.3870 & -0.3854 & -0.3838 & -0.3822 & -0.3806 & -0.3790 & -0.3774 \\
\hline$\left\{\widehat{Y_{1}, Y_{2}}\right\}_{\alpha}$ & $\begin{array}{c}-0.3909 \\
0.0125 \\
0.0002 \\
\end{array}$ & \begin{tabular}{|c|}
-0.3921 \\
0.0186 \\
0.0004 \\
\end{tabular} & \begin{tabular}{|c|}
-0.3891 \\
0.0192 \\
0.0004 \\
\end{tabular} & \begin{tabular}{|c|}
-0.3866 \\
0.0201 \\
0.0004 \\
\end{tabular} & \begin{tabular}{|c|}
-0.3890 \\
0.0267 \\
0.0007 \\
\end{tabular} & \begin{tabular}{|c|}
-0.3806 \\
0.0284 \\
0.0008 \\
\end{tabular} & \begin{tabular}{|c|}
-0.3814 \\
0.0377 \\
0.0014 \\
\end{tabular} & \begin{tabular}{|c|}
-0.3766 \\
0.0447 \\
0.0020 \\
\end{tabular} & \begin{tabular}{|c|}
-0.3747 \\
0.0586 \\
0.0034 \\
\end{tabular} & $\begin{array}{c}-0.3647 \\
0.0743 \\
0.0057 \\
\end{array}$ \\
\hline$\left\{Y_{2}, Y_{1}\right\}_{\alpha}$ & -1.8536 & -1.8899 & -1.9326 & -1.9830 & -2.0421 & -2.1113 & -2.1921 & -2.2858 & -2.3943 & -2.5189 \\
\hline$\left\langle\widehat{Y_{2}, Y_{1}}\right\}_{\alpha}$ & $\begin{array}{c}-1.8620 \\
0.0584 \\
0.0035 \\
\end{array}$ & $\begin{array}{c}-1.8870 \\
0.0815 \\
0.0066 \\
\end{array}$ & \begin{tabular}{|c|}
-1.9343 \\
0.1090 \\
0.0119 \\
\end{tabular} & \begin{tabular}{|c|}
-1.9705 \\
0.0928 \\
0.0088 \\
\end{tabular} & \begin{tabular}{|c|}
-2.0265 \\
0.1225 \\
0.0153 \\
\end{tabular} & \begin{tabular}{|c|}
-2.1182 \\
0.2159 \\
0.0467 \\
\end{tabular} & \begin{tabular}{|c|}
-2.1845 \\
0.2170 \\
0.0472 \\
\end{tabular} & \begin{tabular}{|c}
-2.2987 \\
0.4982 \\
0.2484 \\
\end{tabular} & $\begin{array}{c}-2.3994 \\
0.5074 \\
0.2575 \\
\end{array}$ & $\begin{array}{c}-2.6258 \\
0.8516 \\
0.7367 \\
\end{array}$ \\
\hline $\operatorname{Corr}_{\alpha}$ & 0.7262 & 0.7374 & 0.7510 & 0.7674 & 0.7870 & 0.8103 & 0.8378 & 0.8700 & 0.9074 & 0.9506 \\
\hline$\widehat{\operatorname{Corr}_{\alpha}}$ & $\begin{array}{c}0.7278 \\
0.0302 \\
0.0009 \\
\end{array}$ & $\begin{array}{c}0.7392 \\
0.0347 \\
0.0012 \\
\end{array}$ & $\begin{array}{c}0.7513 \\
0.0320 \\
0.0010 \\
\end{array}$ & $\begin{array}{c}0.7607 \\
0.0322 \\
0.0011 \\
\end{array}$ & $\begin{array}{c}0.7859 \\
0.0353 \\
0.0012 \\
\end{array}$ & $\begin{array}{c}0.8012 \\
0.0338 \\
0.0012 \\
\end{array}$ & $\begin{array}{c}0.8261 \\
0.0335 \\
0.0013 \\
\end{array}$ & $\begin{array}{c}0.8472 \\
0.0371 \\
0.0019 \\
\end{array}$ & $\begin{array}{c}0.8736 \\
0.0402 \\
0.0028 \\
\end{array}$ & $\begin{array}{c}0.9035 \\
0.0381 \\
0.0037 \\
\end{array}$ \\
\hline$r_{S}$ & $\begin{array}{c}-0.8412 \\
0.0139 \\
\end{array}$ & \begin{tabular}{|c|}
-0.8412 \\
0.0145 \\
\end{tabular} & \begin{tabular}{|c|}
-0.8408 \\
0.0151 \\
\end{tabular} & $\begin{array}{c}-0.8420 \\
0.0149 \\
\end{array}$ & $\begin{array}{c}-0.8457 \\
0.0165 \\
\end{array}$ & $\begin{array}{c}-0.8473 \\
0.0166 \\
\end{array}$ & \begin{tabular}{|c|}
-0.8494 \\
0.0172 \\
\end{tabular} & \begin{tabular}{|c|}
-0.8571 \\
0.0161 \\
\end{tabular} & $\begin{array}{c}-0.8607 \\
0.0184 \\
\end{array}$ & $\begin{array}{c}-0.8665 \\
0.0164 \\
\end{array}$ \\
\hline$r_{W}$ & $\begin{array}{c}-0.8505 \\
0.0120 \\
\end{array}$ & $\begin{array}{c}-0.8520 \\
0.0133 \\
\end{array}$ & \begin{tabular}{|c|}
-0.8530 \\
0.0146
\end{tabular} & $\begin{array}{c}-0.8548 \\
0.0138\end{array}$ & $\begin{array}{c}-0.8598 \\
0.0145\end{array}$ & $\begin{array}{c}-0.8608 \\
0.0163 \\
\end{array}$ & $\begin{array}{c}-0.8650 \\
0.0160\end{array}$ & $\begin{array}{c}-0.8726 \\
0.0153\end{array}$ & $\begin{array}{c}-0.8780 \\
0.0159\end{array}$ & $\begin{array}{c}-0.8840 \\
0.0147 \\
\end{array}$ \\
\hline
\end{tabular}




\section{Bibliography}

(1) Samorodnitsky G.; Taqqu M. S. Stable non-Gaussian random processes. Stochastic Modeling. Chapman \& Hall, New York-London, New York, 1994.

(2) Miller G. Properties of certain symmetric stable distributions. Journal of Multivariate Analysis, 1978, 8(3):346-360.

(3) Cambanis S.; Miller G. Linear problems in $p$ th order and stable processes. SIAM Journal on Applied Mathematics, 1981, 41(1):43-69.

(4) Nikias C.; Shao M. Signal Processing with Alpha-Stable Distributions and Applications. Wiley-Interscience, 1995.

(5) Pinkse J. A consistent nonparametric test for serial independence. Journal of Econometrics, 1998, 84(2):205-231.

(6) Tjøstheim D. Measures of dependence and tests of independence. Statistics. A Journal of Theoretical and Applied Statistics, 1996, 28(3):249-284.

(7) Hong Y. Testing for pairwise serial independence via the empirical distribution function. Journal of the Royal Statistical Society. Series B. Statistical Methodology, 1998, 60(2): 429-453.

(8) Hong Y.; White H. Asymptotic distribution theory for nonparametric entropy measures of serial dependence. Preprint, 2003.

(9) Gallagher C. M. A method for fitting stable autoregressive models using the autocovariation function. Statistics $\&$ Probability Letters, 2001, 53(4):381-390.

(10) Chambers J. M.; Mallows C. L.; Stuck B. W. A method for simulating stable random variables. Journal of the American Statistical Association, 1976, 71(354):340-344. 